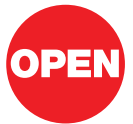

SUBJECT AREAS:

SYNTHESIS AND

PROCESSING

THEORY AND COMPUTATION

MATERIALS FOR DEVICES

MATERIALS CHEMISTRY

Received

20 September 2012

Accepted

19 November 2012

Published

19 December 2012

Correspondence and requests for materials should be addressed to A.M. (manth@austin. utexas.edu)

* These authors contributed equally to this work.

\section{Microwave-assisted Low-temperature Growth of Thin Films in Solution}

\author{
B. Reeja-Jayan ${ }^{1 *}$, Katharine L. Harrison ${ }^{2 *}$, K. Yang ${ }^{3}$, Chih-Liang Wang ${ }^{1}$, A. E. Yilmaz ${ }^{3}$ \\ \& Arumugam Manthiram ${ }^{1,2}$
}

\author{
${ }^{1}$ Materials Science and Engineering Program, The University of Texas at Austin, Austin, Texas 78712, USA, ${ }^{2}$ Mechanical \\ Engineering, The University of Texas at Austin, Austin, Texas 78712, USA, ${ }^{3}$ Electrical and Computer Engineering, The University of \\ Texas at Austin, Austin, Texas 78712, USA.
}

Thin films find a variety of technological applications. Assembling thin films from atoms in the liquid phase is intrinsically a non-equilibrium phenomenon, controlled by the competition between thermodynamics and kinetics. We demonstrate here that microwave energy can assist in assembling atoms into thin films directly on a substrate at significantly lower temperatures than conventional processes, potentially enabling plastic-based electronics. Both experimental and electromagnetic simulation results show microwave fields can selectively interact with a conducting layer on the substrate despite the discrepancy between the substrate size and the microwave wavelength. The microwave interaction leads to localized energy absorption, heating, and subsequent nucleation and growth of the desired films. Electromagnetic simulations show remarkable agreement with experiments and are employed to understand the physics of the microwave interaction and identify conditions to improve uniformity of the films. The films can be patterned and grown on various substrates, enabling their use in widespread applications.

esign and synthesis of new materials is the cornerstone of materials science. In particular, thin film fabrication is important in fields as diverse as semiconductor devices, optoelectronics, energy harnessing/storage, and medicine. Typically, high temperatures are required to synthesize thin films of semiconductor oxides, preventing the use of plastic-based, light-weight, and flexible substrates for solar cells, light emitting diodes, sensors, and photodetectors. Therefore, low-temperature synthesis techniques, which are also facile and energy efficient, are of great interest. A promising avenue to meet these requirements is to exploit solution processes. Thin film growth on substrates in solution is controlled by a competition between thermodynamics and kinetics ${ }^{1-3}$. While particles generally initiate in embryonic nuclei within a solution ("homogeneous nucleation"), thin film growth requires preferential nucleation at interfaces ("heterogeneous nucleation"). Earlier work has shown that microwave radiation can initiate favorable kinetics for thin film growth $^{4,5}$.

Microwave-assisted synthesis is appealing because it can dramatically reduce reaction time, improve product yield, and enhance material properties when compared to conventional synthesis routes ${ }^{6-8}$. While conventional heating is limited by thermal conduction from the vessel walls, microwave fields can quickly and uniformly heat a solution by directly coupling to molecules within the solution through polarization or conduction. Polarization is the process of dipoles formed from bound charges and polar molecules aligning with an oscillating electric field. Conduction is the process of free charge carriers and ions moving in response to an electric field. Collisions resulting from dipole rotation during polarization and charge motion during conduction impart energy to the atoms and molecules in the solution in the form of heat; these two types of heating are known as dielectric and ohmic heating, respectively. Thus, microwave heating can be described using a complex permittivity $\tilde{\varepsilon}$ of the form

$$
\tilde{\varepsilon}=\varepsilon^{\prime}-j\left(\varepsilon^{\prime \prime}+\frac{\sigma}{\omega}\right)
$$

where $\omega$ is the angular frequency of the microwave field and $\varepsilon^{\prime}, \varepsilon^{\prime \prime}$, and $\sigma$ are the permittivity, dielectric loss, and electrical conductivity, respectively. While ohmic loss generally dominates when heating conducting solids, the relative contributions of dielectric loss $\left(\varepsilon^{\prime \prime}\right)$ and ohmic loss $(\sigma / \omega)$ during solution heating depend on the solvent properties, ion concentration, and frequency.

Microwave-assisted heating phenomena are generally attributed to purely "thermal/kinetic" effects, resulting from uniform or rapid heating. However, "specific" and "non-thermal" microwave effects are debated when microwave reaction products differ from conventional synthesis products ${ }^{6}$. Specific microwave effects are defined 
as interactions that are thermal in nature but lead to results that cannot be replicated by conventional heating ${ }^{6}$. The specific microwave effect known as "selective heating" refers to preferential energy absorption by materials with high dielectric/ohmic loss ${ }^{6,9,10}$. A selective heating process is exploited here to cultivate favorable sites for film nucleation; specifically, a highly microwave energy absorbing conducting layer is placed on an insulating substrate within a solution that absorbs weakly relative to the conducting layer, causing selective heating of the conducting layer and enabling thin film assembly. While dielectric/ohmic heating in solution is commonplace in microwave synthesis, selective ohmic heating of solid materials within a growth solution represents a promising new avenue, which has been minimally explored.

To demonstrate the proposed selective microwave-assisted thin film self-assembly, titanium dioxide $\left(\mathrm{TiO}_{2}\right)$ thin films were grown on indium tin oxide (ITO)-coated glass substrates that were immersed in a growth solution and heated in a microwave reactor. $\mathrm{TiO}_{2}$ was chosen for demonstration here because of its diverse applications $\mathrm{s}^{11-13}$. Specifically, the widely sought-after anatase polymorph is used in photodetectors ${ }^{14}$, sensors ${ }^{15}$, dye-sensitized solar cells ${ }^{16}$, photocatalytic water splitting ${ }^{17}$, hybrid polymer solar cells ${ }^{18}$, and lithium-ion battery anodes $^{19,20}$. We show that the ITO layer strongly absorbs microwave energy, causing localized heating that catalyzes growth of anatase $\mathrm{TiO}_{2}$ thin films while the solution temperature remains at $150^{\circ} \mathrm{C}$. In contrast, classical synthesis routes for anatase films comprise of chemical deposition techniques (sol-gel) and vacuum deposition techniques (sputtering, atomic layer deposition), followed by a high-temperature sintering step at $\geq 450^{\circ} \mathrm{C}$ to crystallize the films ${ }^{11}$. Such high temperatures limit the choice of thin film growth substrates as flexible polymeric/plastic substrates typically decompose between 100 and $300^{\circ} \mathrm{C}^{21}$. Differences between the conventional and the proposed microwave-assisted synthesis routes are illustrated in Fig. 1.

Further details on the microwave-assisted synthesis scheme are shown in Fig. 1 and presented subsequently. A microwave reactor (Anton Paar Synthos 3000) operating at $2.45 \mathrm{GHz}$ was used for reproducible control and monitoring of temperature, pressure, and power. Glass substrates coated with square shaped ITO regions (blue square in Fig. 1) were placed inside custom designed glass baskets that were hung from the top of quartz vessels containing a mixture of a sol-gel based Ti precursor and tetraethylene glycol (TEG) ${ }^{14}$. The vessels were sealed to allow autogenous pressure to build under solvothermal reaction conditions. The microwave heating process can be described as follows: the walls of the quartz vessels do not absorb significant microwave energy, allowing the solution to be heated directly by dielectric and ohmic mechanisms ${ }^{6-8}$. The ITO layer (conductivity $\sigma \sim 10^{5} \mathrm{~S} / \mathrm{m}$ ) also absorbs microwave energy (predominantly by ohmic heating) and it does so more efficiently than the solution, creating a site for $\mathrm{TiO}_{2}$ to nucleate and grow in a single step. As seen in Fig. 1, no film forms on the glass, which also does not absorb significant energy and remains cooler than the ITO layer.

\section{Results}

Growth and characterization of $\mathrm{TiO}_{2}$ thin films on various substrates. The microwave-assisted film growth process was run on ITO-coated glass under a wide range of conditions to optimize uniformity and crystallinity of the $\mathrm{TiO}_{2}$ films. A summary of the key results, shown in Fig. 2a, reveals that the best film growth occurs at the convergence of optimum temperature and reaction time, but the power ramp rate is not a critical variable. The films grown at $150^{\circ} \mathrm{C}$ followed by a $60 \mathrm{~min}$ reaction time appear most uniform. The films are thin at low temperatures and short reaction times, but higher temperatures and longer times cause chunks of film to peel off as the films grow too thick. At higher reaction temperatures, shorter reaction times can be used to obtain thinner films. Optimum film growth was dependent upon the use of TEG. More details regarding synthesis optimization are discussed under Supplementary Section 1. Films grown under optimized conditions are strongly adhered likely owing to oxide-oxide bonding between $\mathrm{TiO}_{2}$ and ITO. Sonication for $10 \mathrm{~min}$ in various solvents does not damage the films. Unlike in conventional film growth techniques, the conductivity of the ITO
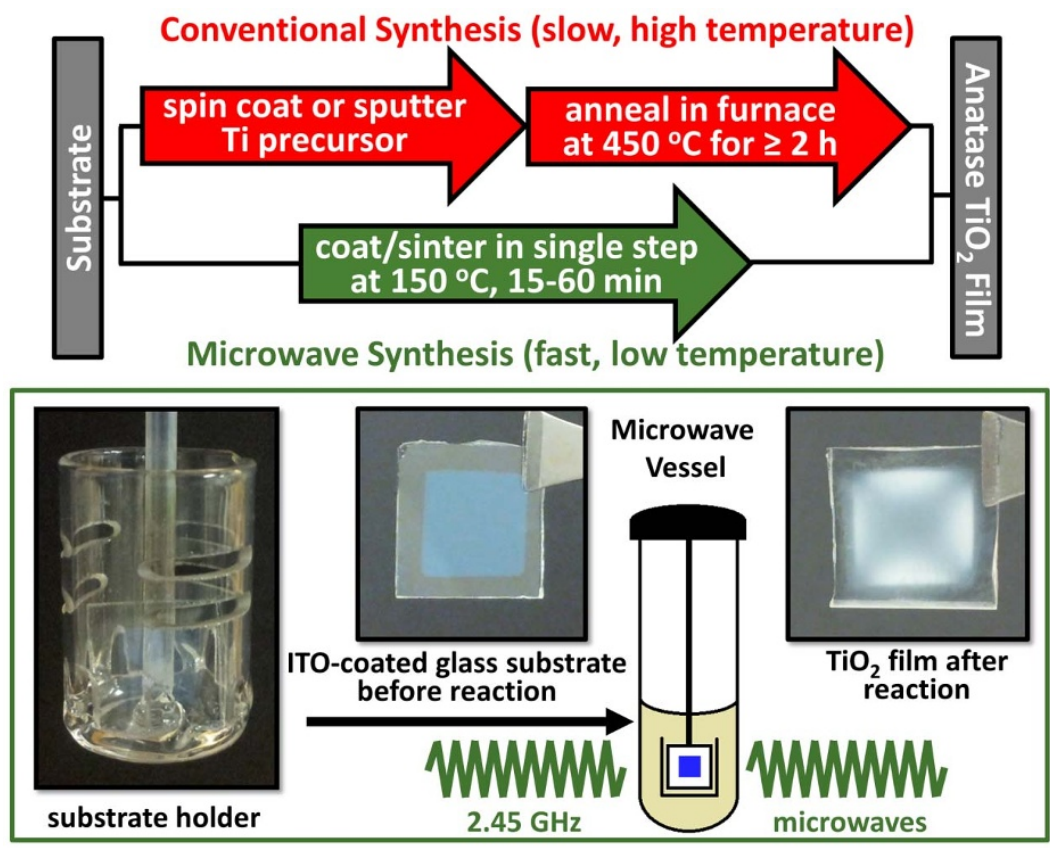

Figure 1 Comparison of conventional and microwave-assisted solvothermal $\mathrm{TiO}_{2}$ thin film growth processes on indium tin oxide (ITO)-coated glass substrates (top) and a schematic of the microwave reaction scheme (bottom). Quartz microwave vessels were secured in an Anton Paar Synthos 3000 microwave reactor and the substrates were suspended from the caps with custom designed glass baskets (shown bottom left). The pale yellow growth solution consisted of tetraethylene glycol (TEG) and a Ti-based sol-gel precursor. The conducting ITO layer absorbs microwave radiation and heats, creating a nucleation site for the $\mathrm{TiO}_{2}$ film to grow. 
layer does not decrease during the microwave-assisted film growth process.

The crystallinity of the microwave-grown films was studied with glancing incidence X-ray diffraction (GIXRD) and Raman spectroscopy. Microwave interaction yields crystalline films starting at temperatures as low as $140^{\circ} \mathrm{C}$. Sharper anatase peaks appear at $150^{\circ} \mathrm{C}$, as shown in Fig. 2b, which compares the GIXRD patterns of the microwave-grown films with those of conventionally-grown films on ITOcoated glass. Both GIXRD patterns show the appearance of a strong anatase (101) peak at $25^{\circ}$, indicating that the microwave-assisted process results in crystalline, anatase $\mathrm{TiO}_{2}$ films. Fig. 2c-e show microwave deposited $\mathrm{TiO}_{2}$ films on ITO-coated glass, metal (aluminum)-coated glass, and ITO-coated plastic (polyethylene terephthalate $(\mathrm{PET})$ ) substrates, demonstrating that the microwave-assisted film growth process can be adapted to various substrates.

The morphology of the microwave-grown $\mathrm{TiO}_{2}$ films was characterized by several techniques spanning a wide range of length scales. Scanning transmission electron microscopy (STEM) image in Fig. 3a reveals that these films are comprised of aggregates of 15-20 nm crystallites. High-resolution transmission electron microscopy (TEM) imaging (Fig. 3a inset) of these crystallites indicate crystalline fringes with a d-spacing of $0.35 \mathrm{~nm}$, corresponding to the (101) plane of anatase $\mathrm{TiO}_{2}$. Large area scanning electron microscopy (SEM) images (Fig. 3b and 3c) reveal a smooth morphology, indicating that the films are dense and continuous unlike the loosely bound particles which have been shown previously ${ }^{4,5}$. Further char- acterization with atomic force microscopy (AFM) confirms the STEM findings that the microwave-grown $\mathrm{TiO}_{2}$ films are comprised of 100-200 nm self-sintered grains that are aggregates of smaller crystallites (Fig. 3d). Conductive AFM (C-AFM) imaging was used to simultaneously map the topography of the microwave-grown $\mathrm{TiO}_{2}$ films along with areas of varying conductivity. The current map (Fig. 3f) tracks the topography map (Fig. 3e). These images reveal that the microwave-grown $\mathrm{TiO}_{2}$ films are uniformly insulating, but the central part of the film shows some current leakage.

The thicknesses of the optimized films are approximately $2000 \mathrm{~nm}$ (thinnest in the middle and thicker at the edges). The samples heated at lower temperatures or shorter times are found to be thinner, and the samples heated at higher temperatures and longer times are thicker. Orientation of the substrate inside the reaction vessel also has a dramatic effect on film growth. GIXRD patterns show significantly stronger anatase peaks for films grown in the vertical orientation. This observation agrees with visual inspection of the films and cross-sectional SEM images, indicating that thinner films grow in the horizontal orientation $(200 \mathrm{~nm})$ than in the vertical orientation $(2000 \mathrm{~nm})$. This variation suggests stronger interaction between the microwave fields and the ITO layer when the latter is placed in a vertical orientation. Cross-sectional SEM images are depicted under Supplementary Sections 1-2.

The results in Figs. $2 \mathrm{~b}$ and $3 \mathrm{a}$ show that crystalline anatase $\mathrm{TiO}_{2}$ films can be grown at a significantly lower temperature $\left(150^{\circ} \mathrm{C}\right)$ compared to conventional techniques $\left(450^{\circ} \mathrm{C}\right)$. It is important to
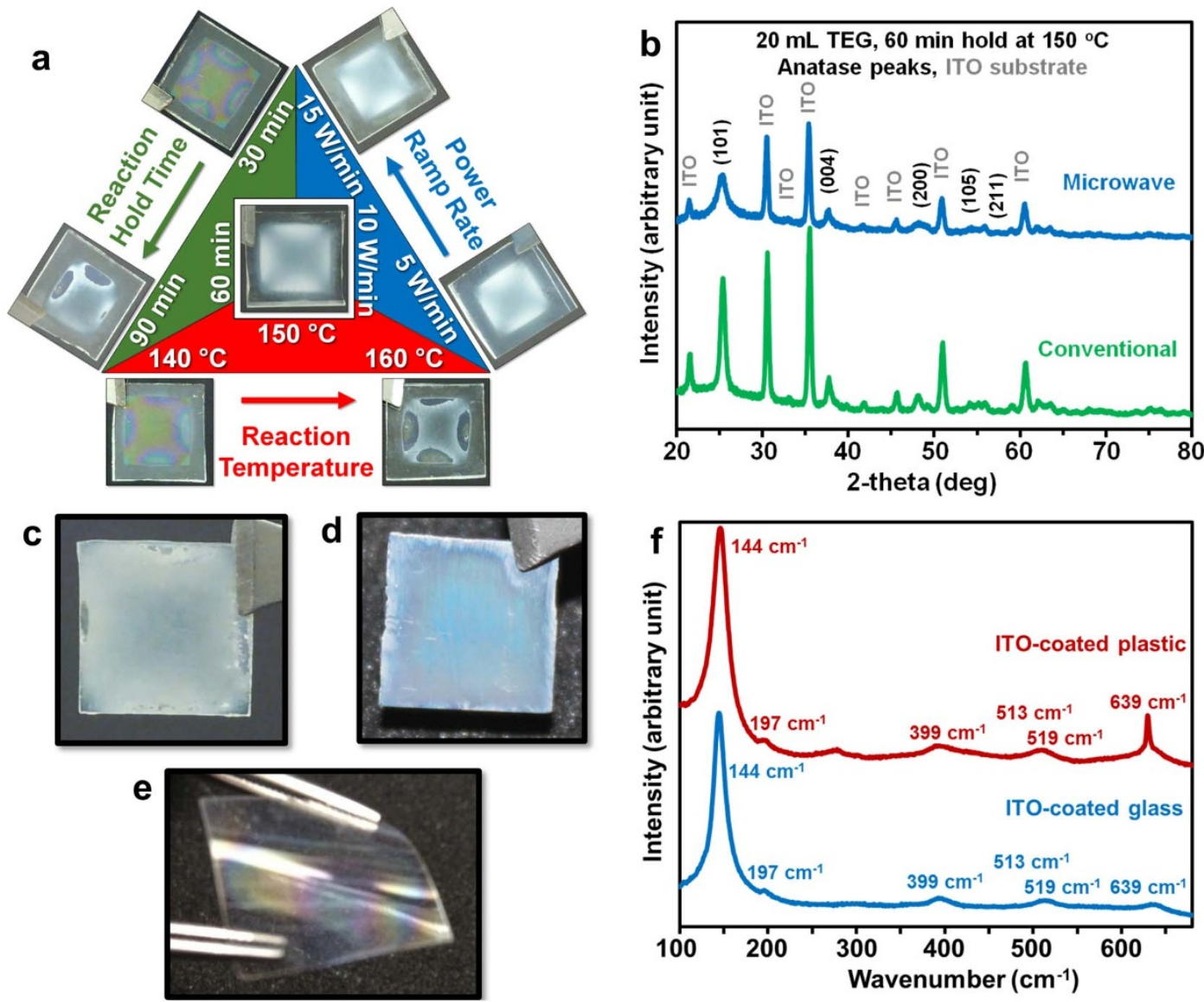

Figure $2 \mid$ Microwave-assisted solvothermal synthesis results for anatase $\mathrm{TiO}_{2}$ thin films. (a) Summary of film growth optimization conditions. The most uniform and thickest films were grown at a temperature of $150^{\circ} \mathrm{C}$ held for 60 minutes. Shorter hold times or lower temperatures led to thinner, more amorphous films. Longer times or higher temperatures led to film delamination at the edges. The power ramp rate had little effect on film formation. (b) GIXRD pattern for the optimized film grown on ITO-coated glass shows peaks for the ITO layer as well as strong anatase phase peaks, most notably the (101) peak at $25^{\circ}$. (c) Microwave-grown $\mathrm{TiO}_{2}$ film on ITO-coated glass. (d) Microwave-grown $\mathrm{TiO}_{2}$ film on Al-coated glass. (e) $\mathrm{Microwave}$-grown TiO 2 film on ITO-coated PET. (f) Raman spectroscopy for films grown on ITO-coated glass and ITO-coated PET. 

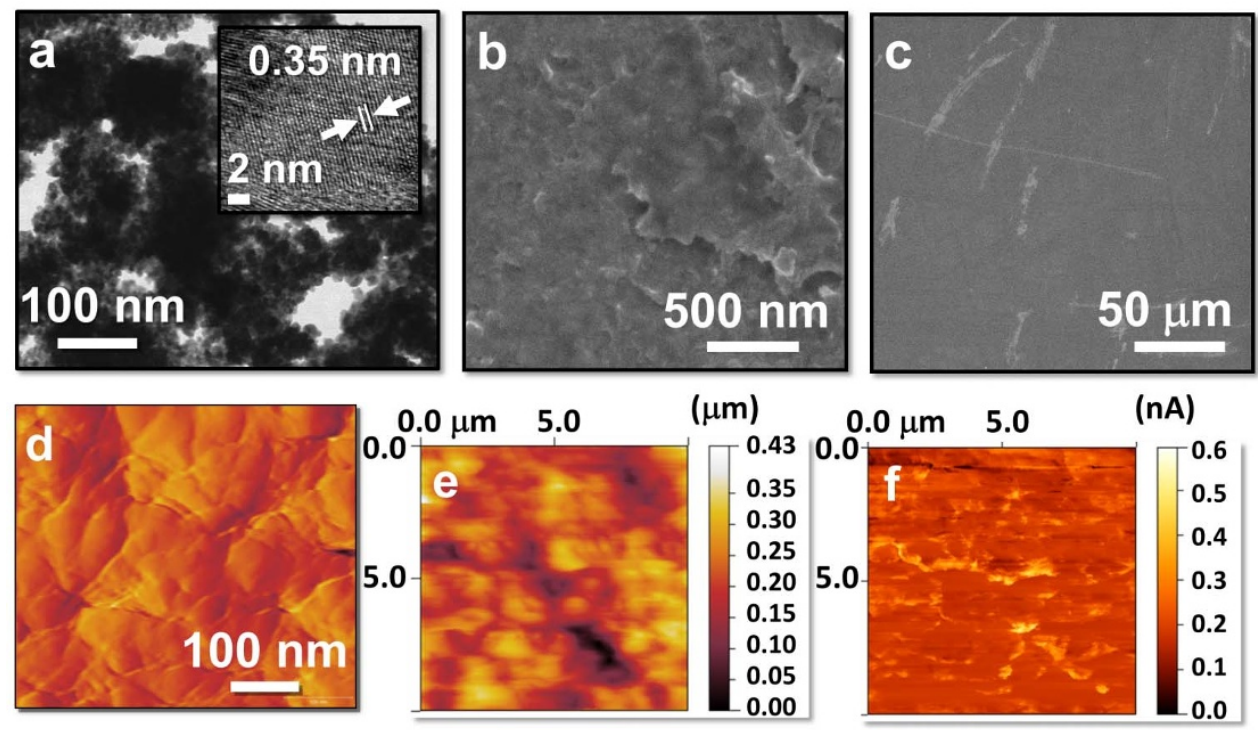

Figure $3 \mid$ Morphology and electrical characterization for a $\mathrm{TiO}_{2}$ film grown on ITO-coated glass at $150^{\circ} \mathrm{C}$ for a 60 min reaction time inside a microwave reactor with a $10 \mathrm{~W} / \mathrm{m}$ power ramp rate. (a) Scanning TEM image indicates 15-20 nm crystallites. High-resolution TEM (inset) confirms the presence of crystalline anatase. (b) Magnified SEM image of the film. (c) Large area SEM image of the film. (d) AFM image showing 100-200 nm selfsintered $\mathrm{TiO}_{2}$ grains. (e) Conductive AFM topography map. (f) Conductive AFM current map.

observe that the temperature values reported here are the average solution temperatures, which are the only temperatures that can be measured during the microwave reaction. In fact, different parts of the ITO layer, the substrate, and the solution can be at different temperatures during the reaction because of their different rates of microwave energy absorption. Specifically, it should be expected that parts of the ITO layer, which selectively absorbs microwaves, and parts of the glass substrate and solution that are in thermal contact with the ITO layer are (temporarily) at higher temperatures than the average solution temperature. If the temperatures locally become much higher than the average solution temperature (and remain so for long durations), film growth would be hindered on temperature sensitive substrates. However, it can be deduced that the temperature of the substrate during the microwave-assisted film growth process cannot be significantly higher than the solution temperature of $150^{\circ} \mathrm{C}$, because we are able to grow $\mathrm{TiO}_{2}$ films on plastic (PET) substrates, which have a melting temperature of $250^{\circ} \mathrm{C}^{21}$, without melting or deforming them (Fig. 2e). The formation of anatase phase cannot be confirmed by GIXRD for $\mathrm{TiO}_{2}$ films grown on plastic substrates, as PET presents a peak that overlaps with the strongest anatase (101) peak at $25^{\circ}$. Accordingly, Raman spectroscopy analysis was used to compare $\mathrm{TiO}_{2}$ films grown on ITO-coated plastic substrates to the films grown on ITO-coated glass. As indicated in Fig. 2f, Raman analysis of both films show six Raman active modes attributed to anatase $\mathrm{TiO}_{2}$. The peaks seen at 280 and $630 \mathrm{~cm}^{-1}$ in Fig. $2 \mathrm{f}$ can be attributed to the PET substrate. Absence of additional peaks suggests that there are no significant impurities leftover from the microwave reaction at $150^{\circ} \mathrm{C}^{22}$. Thus, Raman analysis confirms that crystalline anatase films grow on the plastic substrate, establishing clearly that the microwave-assisted process can be used to grow crystalline films on plastic or temperature sensitive substrates.

Mechanism of film growth. Although "thermal effects" like volumetric heating caused by microwave interactions with solutions are well understood, "specific effects" like the selective heating of ITO described here are considered speculative ${ }^{6}$. As shown in Fig. 1, the microwave-grown $\mathrm{TiO}_{2}$ films are thicker at the edges compared to the center, giving them a clover or X-like appearance. This pattern changes depending on the size, orientation, conductivity of the ITO layer, and type of microwave equipment (Supplementary Section 3) used. Such edge patterns may be surprising, because $2.45 \mathrm{GHz}$ microwaves comprise of waves with a wavelength of $\sim 12 \mathrm{~cm}$ in air, which are too large to cause any distinguishable incident electric field distribution on the small substrates used here. Indeed, the electric fields must concentrate at the edges of the ITO layer, because of the so-called "edge condition." The edge condition can be used to show analytically that for a perfectly conducting surface with sharp edges, the electric and magnetic field components normal to the edge become infinite at the edge ${ }^{23,24}$. For materials with finite conductivity, like the ITO layer $\left(\sigma \sim 10^{5} \mathrm{~S} / \mathrm{m}\right)$, the field components normal to sharp edges remain finite. Higher conductivities lead to concentrated fields at the edges, resembling the perfectly conducting surface.

To better understand the microwave-assisted selective heating of the ITO layer and the resulting film growth, an electromagnetic model of the experimental setup was constructed. The model consisted of the rectangular cavity of the microwave reactor, the metallic parts of the microwave rotor, the quartz vessel, the solution, the glass substrate, and the ITO layer as detailed in Supplementary Section 4. It was found that all these components, which impose additional boundary conditions on electromagnetic fields and thus change the field distribution throughout the microwave cavity, had to be modeled to perform predictive simulations. For instance, initial models that did not include the conducting parts of the rotor, which scatter microwave fields strongly, did not yield satisfactory results. Fig. 4 shows some of the experimental elements that were simulated; specifically, it shows the field distribution on the microwave rotor and the ITO-coated glass substrate for one of the simulated positions of the vessel in the cavity. Because of the complexity of the model, the microwave energy absorption was analyzed using an advanced simulator that solves surface and volume integro-differential equations for electromagnetic fields subject to appropriate boundary conditions. The fields calculated by these simulations are used to determine the microwave energy absorbed by the ITO layer. Specifically, the absorbed energy density (e), which is a point-wise function that quantifies the amount of incident electromagnetic energy converted to thermal energy at each point, is computed per one full rotation of the substrates in the microwave reactor (see equations (10)-(12) in Supplementary Section 4) and the results are visualized in Fig. 5i-1. The figures show that the energy absorbed is null (dark blue) on the glass portion of the substrate, which is modeled as a lossless (non- 


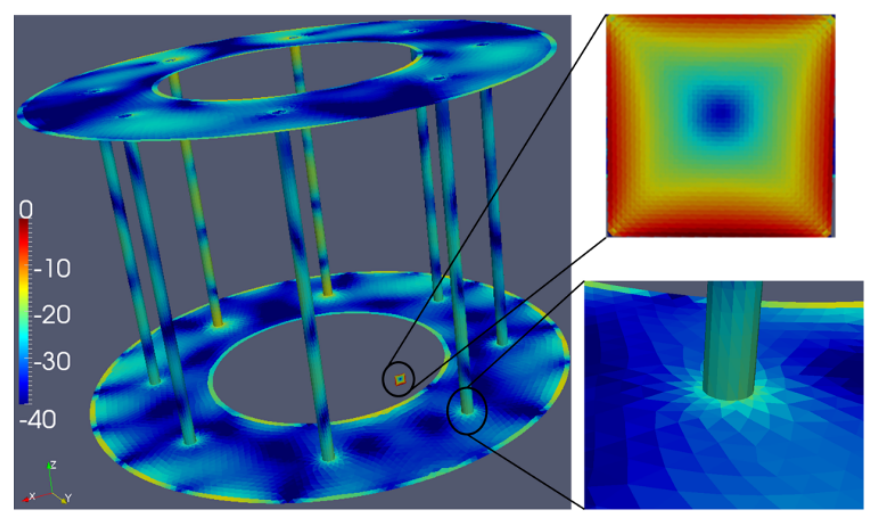

Figure $4 \mid$ Simulated microwave fields on the microwave rotor and the ITO-coated glass substrate. The magnitude of the surface current densities $\mathbf{J}^{\mathrm{PEC}}$ and $\mathbf{J}^{\mathrm{TDS}}$ (defined in Supplementary Section 4) are computed for one simulated position. The current densities are normalized by their maximum magnitude and the color bar is in $\mathrm{dB}$ scale.

absorbing) dielectric, the highest (yellow to red color) on the edges, and intermediate (light blue) at the center of the ITO.

The simulated data show that the electromagnetic energy absorption is concentrated at the edges of the ITO resulting in thicker $\mathrm{TiO}_{2}$ films in these regions, irrespective of the ITO pattern shape. The experimentally observed edge patterns resulting from the concentration of the $\mathrm{TiO}_{2}$ films at the ITO edges show strong correlation to the simulations (Fig. 5). Although heat transfer is not included in the simulations, it is straightforward to reason that strongly absorbing regions will have elevated temperatures, catalyzing nucleation and leading to thicker film growth. Heat may then diffuse inward, leading to further film growth all over the ITO, albeit slightly thinner in the middle.

An important advantage of the proposed microwave-assisted selective heating process is the ability to pattern the $\mathrm{TiO}_{2}$ films in situ as opposed to conventionally grown films which have to be patterned after synthesis. As seen in Fig. 5, patterning the catalyst ITO layer prior to synthesis patterns the microwave-grown $\mathrm{TiO}_{2}$ film. Film thickness variations present an obstacle for growing large areas of uniform $\mathrm{TiO}_{2}$ films by the microwave-assisted process. Fortunately, electromagnetic simulations predict that uniform films can be grown by decreasing the conductivity of the ITO layer (Fig. 6a).

Role of conducting layer on microwave absorption. Conductivity $(\sigma)$ of the microwave absorbing layer plays an important role in the microwave interaction ${ }^{25}$. As seen in Fig. 6a, the total microwave energy absorbed by the conducting layer increases as the conductivity increases, until a point is reached where the trend reverses. This is because, as $\sigma$ increases, more energy is reflected rather than absorbed by the conducting layer, i.e., the electric field magnitude $(|\mathbf{E}|)$ decreases in the conducting layer while $\sigma$ increases and hence the absorbed energy density $e$, which is the time integral of $\sigma|\mathbf{E}|^{2}$, and the total absorbed energy by the conducting layer decreases. Thus, there exists a range of $\sigma$ values where energy absorption is maximized and, therefore, would be ideal for film growth. Experimentally, we find that while films did not grow on insulators like glass, they grow well on ITO, which has a reasonably high conductivity $(\sigma \sim$ $10^{5} \mathrm{~S} / \mathrm{m}$ ). Conversely, if conductivity is too high such as for an aluminum substrate $\left(\sigma \sim 10^{7} \mathrm{~S} / \mathrm{m}\right)$, the microwave-grown films are more weakly adhered and amorphous. While films grown on ITO are highly optimized, films grown on metal require further optimization. The electromagnetic simulations further indicate that at lower conductivity, the films tend to be more uniform compared to those at higher conductivity (insets in Fig. 6a). Based on the computational predictions that lower ITO conductivity can improve film uniformity, $\mathrm{TiO}_{2}$ films were also grown on lower conductivity $(\sigma$ $\sim 10^{3} \mathrm{~S} / \mathrm{m}$ ) ITO, and the film uniformity was found to be greatly improved (Fig. 6c) in agreement with the simulations.
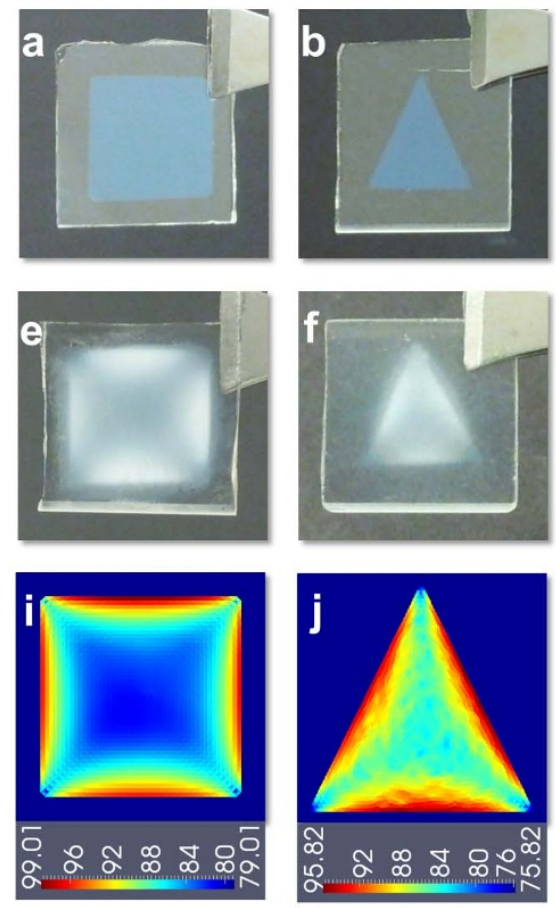
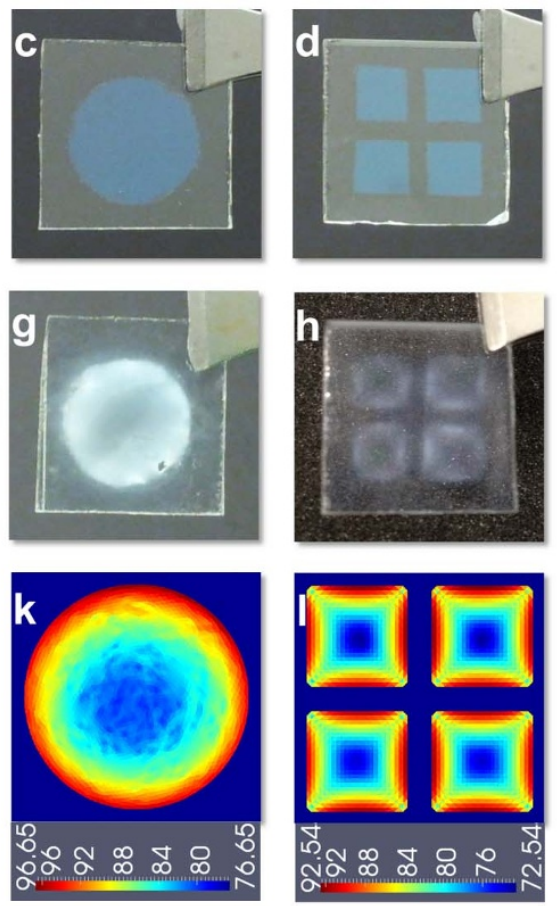

Figure $5 \mid \mathrm{TiO}_{2}$ thin films grown on ITO-coated glass compared to the simulated microwave energy absorption. (a-d) Patterned catalyst ITO layers. (e-h) $\mathrm{TiO}_{2}$ films grown on ITO by selective heating after the microwave reaction. These films were all grown by microwave reaction with a $10 \mathrm{~W} / \mathrm{min}$ power ramp rate to $150^{\circ} \mathrm{C}$ with a $60 \mathrm{~min}$ hold time, except for the film in (h) which was held at $170^{\circ} \mathrm{C}$ for $60 \mathrm{~min}$. (i-l) The absorbed energy density per rotor rotation extracted from electromagnetic simulations. The energy densities are normalized by $1 \mathrm{~J} / \mathrm{m}^{3}$ and the color bars are in $\mathrm{dB}$ scale. The computational and experimental results show strong correlation. 

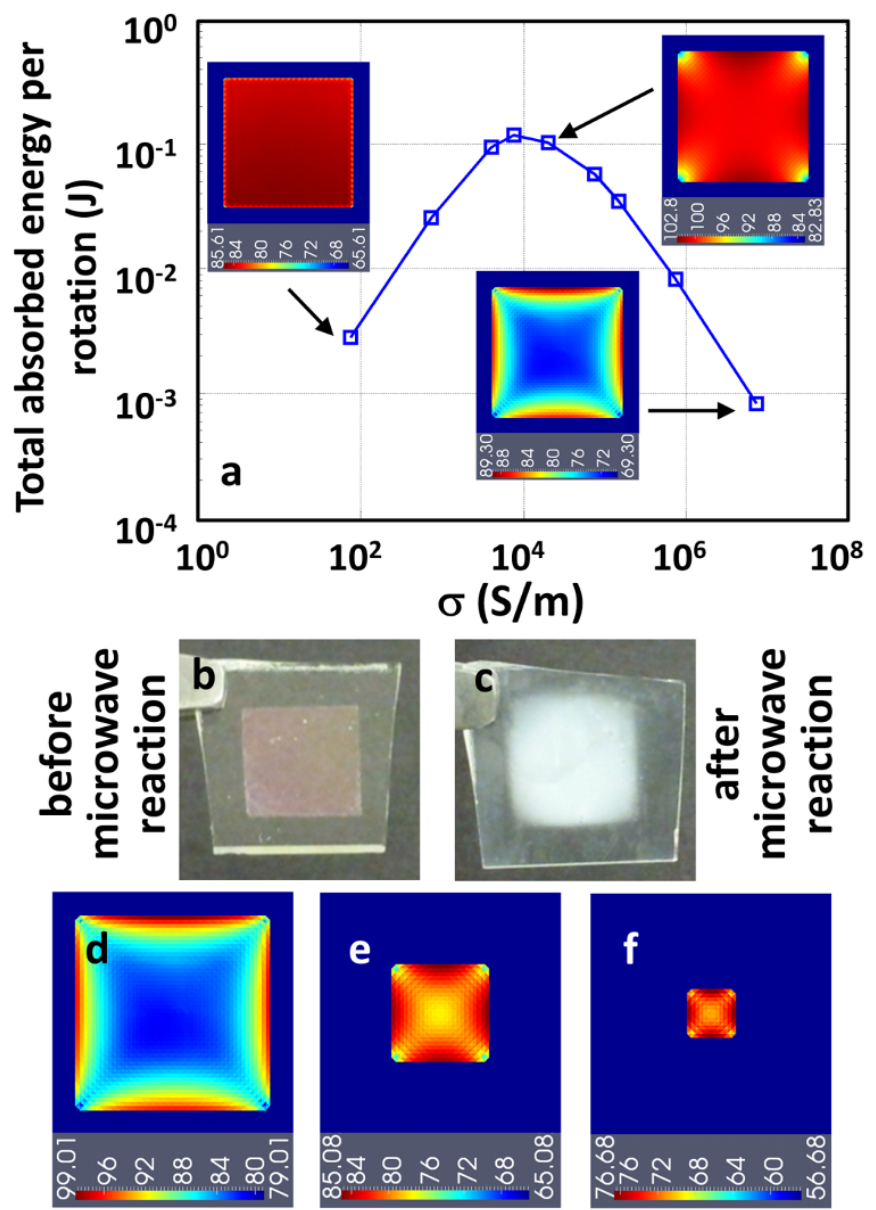

Figure $6 \mid$ Effects of ITO layer conductivity and size on the total absorbed microwave energy and on absorption patterns. (a) Total microwave energy absorbed by the ITO layer per rotor rotation as the layer conductivity is changed with local absorption patterns (insets). Lower conductivity $\left(\sigma \sim 10^{3} \mathrm{~S} / \mathrm{m}\right)$ ITO-coated glass substrate (b) before microwave reaction and (c) after microwave reaction. Films grown on lower conductivity ITO layers do not exhibit edge patterns and are more uniform than those shown in Fig. 5. Absorbed energy density per rotor rotation for ITO layer pattern sizes of (d) $0.8 \mathrm{~cm} \times 0.8 \mathrm{~cm}$, (e) $0.4 \mathrm{~cm} \mathrm{x}$ $0.4 \mathrm{~cm}$, and (f) $0.2 \mathrm{~cm} \times 0.2 \mathrm{~cm}$. The energy densities are normalized by $1 \mathrm{~J} / \mathrm{m}^{3}$ and the color bars are in $\mathrm{dB}$ scale. These results suggest that decreasing the ITO pattern conductivity or size can improve film uniformity.

It must be mentioned that the thickness $(t)$ of the ITO layer, which was $\sim 150 \mathrm{~nm}$ for the results presented here, also affects the film growth process. For relatively thin $(<1 \mu \mathrm{m})$ layers of ITO, the electric field magnitude and the absorbed energy density inside the layer are essentially constant with respect to the direction normal to the ITO surface, i.e., they are uniform throughout the thickness. In this case, the product $\sigma t$ determines the surface impedance of the layer (see equation for $Z_{T D S}$ in Supplementary Section 4). Thus, for a fixed value of $\sigma t$, the electric field magnitude in the ITO layer, the total amount of microwave energy absorbed by the layer, the pattern of the microwave absorption, and the uniformity of the films grown will be essentially identical. However, the smaller the $\sigma$ and the larger the $t$ are for a fixed value of their product, the smaller the absorbed energy density will be and the lesser the ITO layer will heat up relative to the solution, which implies that the input microwave power will have to be increased. Otherwise, changing the ITO layer thickness will not affect the conclusions presented here as long as $\sigma t$ is fixed. As the ITO layer becomes thicker, the absorbed energy density will no longer be uniform throughout the thickness and both the experimental results and the modeling assumptions will change, particularly as $t$ approaches the microwave skin depth $(\sim 11 \mu \mathrm{m})$. Detailed investigation of the effect of conducting layer thickness on the microwave-assisted film growth process is planned for future study.

Decreasing the size of the ITO layer can also improve film uniformity. The microwave-assisted process is optimized for ITO patterns with a feature size $\sim 1 \mathrm{~cm}$ (Fig. 5a-c). Attempts to reduce these dimensions by half result in no film growth even when the total ITO area is kept constant by patterning four features onto the substrate (Fig. 5d). The microwave input power has to be significantly increased (which equates to a higher solution temperature of about $170^{\circ} \mathrm{C}$ ) in order to obtain films on smaller ITO regions. If the dimension of the ITO pattern is comparable to the wavelength $(\lambda)$ in the solution, large area films of $\mathrm{TiO}_{2}$ are selectively deposited on the ITO (Fig. 6d). Conversely, as the dimensions of the ITO patterns become smaller compared to $\lambda$, the microwave fields cannot interact as effectively with the microwave absorbing layer. This results in lowering the overall energy absorption and subsequent heating, thereby requiring higher microwave power for the films to form. This trend is confirmed by simulations which show that the absorbed energy density is smaller when ITO features are smaller (Fig. 6e and f). Such dependence of the film growth conditions on the dimensions of the ITO features is yet another indicator of a "specific" microwave effect.

Selective microwave heating. Further evidence that the film growth is dependent upon selective microwave heating of the ITO layer can be demonstrated experimentally. Additional experiments were run in an Anton Paar Monowave microwave in both glass and silicon carbide $(\mathrm{SiC})$ vessels with otherwise identical heating conditions (Fig. 7). The ramp time, heating temperature, and reaction hold time were the same in both reactions. The only difference was the material of the vessel. Fig. 7 shows that no film forms when the reaction is run in the $\mathrm{SiC}$ vessel, while a film successfully forms in

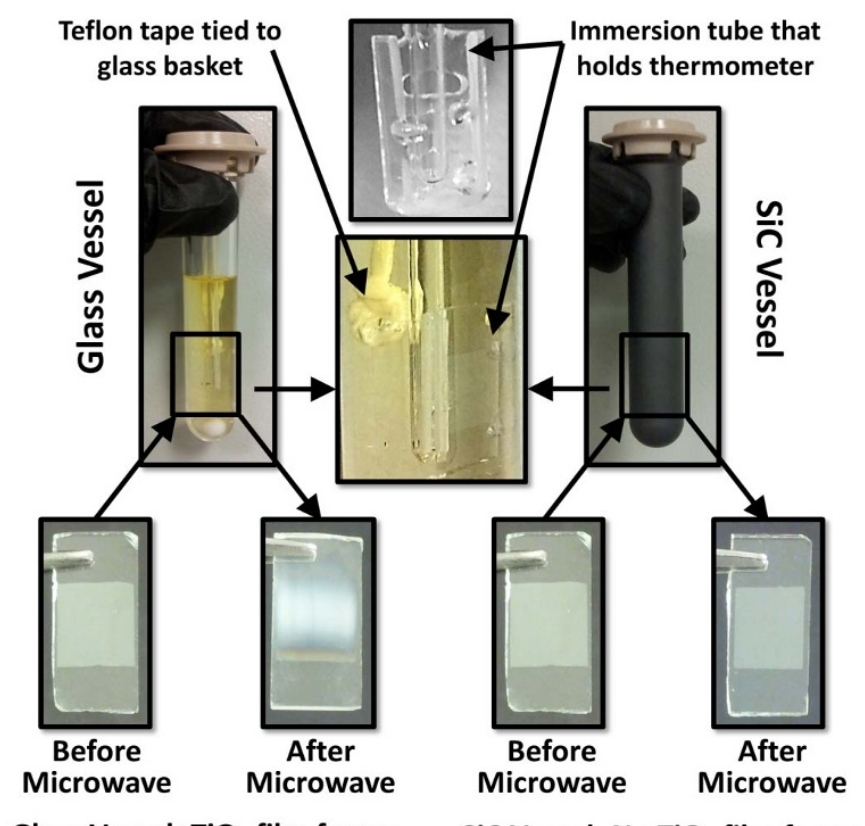

Glass Vessel: $\mathrm{TiO}_{2}$ film forms

SiC Vessel: $\mathrm{No}_{\mathrm{TiO}_{2}}$ film forms

Figure 7 | Comparison of ITO substrates before and after microwave reactions in glass and $\mathrm{SiC}$ vessels for reactions ramped to $160^{\circ} \mathrm{C}$ in $3 \mathrm{~min}$ and held for 60 minutes. The reactions were run with the same conditions except for the vessel material. Only reactions in the glass vessel led to film formation because $\mathrm{SiC}$ shields the ITO from direct interaction with the microwaves. 
the glass vessel. We ran these reactions with different ramp times and temperatures, and were never able to grow films in the $\mathrm{SiC}$ vessel. In contrast, we were able to grow films at a variety of temperatures and ramp times in the glass vessel.

Glass is essentially transparent to microwaves; consequently, when an ITO-coated glass substrate is suspended within the $\mathrm{TiO}_{2}$ growth solution in a glass vessel, the microwave field can interact directly with the solution and ITO layer, allowing them to heat by microwave absorption. In this case, as in the above experiments, heating occurs by selective ohmic heating of the ITO layer and dielectric/ohmic heating of the solution. In contrast, because $\mathrm{SiC}$ is a strong microwave absorber, the walls of the $\mathrm{SiC}$ vessel absorb the microwave energy effectively and prevent microwave absorption by the solution and ITO layer ${ }^{26}$. Here, heating occurs through microwave absorption by the $\mathrm{SiC}$ (ohmic heating) and subsequent thermal convection through the solution. This causes the ITO layer to be heated indirectly by thermal convection in the $\mathrm{SiC}$ vessel rather than by direct selective microwave heating. As a result, the ITO layer does not selectively absorb microwaves in the $\mathrm{SiC}$ vessel and cannot act as a hot nucleation site for heterogeneous film formation. Note that a common argument used to dismiss specific microwave effects is that the conditions during microwave heating, such as the temperature ramping time, are difficult to replicate by conventional methods $\mathrm{o}^{6,26,27}$. In these experiments, however, even poorly microwave absorbing solutions can be rapidly heated by thermal convection in the $\mathrm{SiC}$ vessel because of the small size of the vessel $(10 \mathrm{~mL})$ and the high power available from the Monowave. These experiments further confirm that film growth is critically dependent on the selective heating of the ITO layer which results from direct interaction with the microwave field.

Conventional solvothermal synthesis was also attempted by placing ITO-coated glass substrates in pressure vessels and heating in a furnace at various temperatures and times $\left(150-180^{\circ} \mathrm{C}\right.$ and $\left.2-72 \mathrm{~h}\right)$. These experiments resulted in no film growth even though anatase $\mathrm{TiO}_{2}$ formed in solution. This result reiterates that the microwave interaction with the conducting layer is necessary for film growth to occur. More details regarding the $\mathrm{SiC}$ experiments are shown in Fig. 7 and the conventional solvothermal experiments are discussed in Supplementary Sections 5 and 6.

\section{Discussion}

The ability to produce crystalline thin films of oxide materials like anatase $\mathrm{TiO}_{2}$ at low temperatures has tremendous commercial advantage for thin film electronics. To demonstrate this, microwave-grown $\mathrm{TiO}_{2}$ films were incorporated into photodetectors, hybrid solar cells, and lithium-ion batteries. Although these prototypes are not optimized, they demonstrate that the microwavegrown films have good crystallinity and can be successfully used in thin-film device applications (Supplementary Section 7). Electromagnetic simulations may prove helpful for optimizing film growth, thereby improving the device performance. Microwavegrown films may additionally find application in other areas including ultracapacitors, dye-sensitized solar cells, hydrogen production, photocatalytic water splitting, functional biomaterials, medical devices, and self-cleaning/anticorrosion coatings.

Microwave energy is generally simply considered a heat source that leads to faster chemical reactions and better product yield and purity than conventional furnace heating. It is usually challenging to prove definitively that selective microwave heating is responsible for changes seen between conventional and microwave-assisted heating. Here we have shown clearly through the $\mathrm{SiC}$ vessel experiments and electromagnetic simulations that selective microwave absorption by the conducting (ITO) layer on the substrate is necessary for film growth to occur. This result is important because if the film growth is dependent on direct interaction between the microwave field and the conducting layer, it is possible to adjust microwave parameters such as power, frequency, and geometry to allow more control over film growth than one can achieve by simply varying solution parameters such as reaction time and temperature.

The excellent agreement between our experiments and the electromagnetic simulations is evidence that these simulations can be used to predict the suitability of various conducting layers with a given size and shape for obtaining uniform films. This is advantageous because while ITO is an appropriate conducting layer for certain types of solar cells ${ }^{16,18}$, other conducting layers would be required for other applications. The electromagnetic simulations further reveal that there exists an optimum range for conductivity (and thickness) of the microwave absorbing layer for sufficient absorption of the microwave radiation and that there is a defined relationship between the size of the conducting layer and microwave absorption pattern (and resulting film uniformity) for a given microwave frequency. It is also interesting that as the ITO pattern dimension shrinks to length scales significantly smaller than the wavelength, the films grow all over the substrate, bridging the gaps between ITO regions (Supplementary Section 8). This bridging effect is comparable to the welding effect reported when microwave irradiation produced fusion of Ag nanowires in ethylene glycol into nanowire networks ${ }^{6,28}$. A practical application of this effect would be to design micro-patterned electrodes to concentrate the microwave energy into hot spots acting as microwave tweezers, which could assemble films without a continuous ITO layer. Future experiments will exploit this concept to eliminate the need of a continuous microwave absorbing layer to catalyze film growth. Growing such tailored films also promises future directions in plasmonics and biomedical applications.

In summary, low-temperature, solution-based processes must be developed to grow thin films on temperature sensitive substrates such as plastic. Growing thin films in solution requires providing the atoms an incentive to grow on a substrate rather than heterogeneously in solution. This work demonstrates that microwave absorption by a conducting layer on a substrate can provide such an incentive via selective heating. Specifically, we have used the microwave-assisted, low-temperature method to grow thin films of anatase $\mathrm{TiO}_{2}$ at $150^{\circ} \mathrm{C}$, a significant decrease from the $450^{\circ} \mathrm{C}$ required for conventional film growth. The films are crystalline as demonstrated by GIXRD, Raman, and TEM analysis. We show that the proposed microwave-assisted process is highly versatile and can grow thin films of anatase $\mathrm{TiO}_{2}$ on conducting layers located on various substrates including plastic (PET), a promising result for plastic-based electronics. Films grown by the microwave-assisted process can also be neatly patterned in situ, while the conducting layer remains intact, enabling their use in widespread applications. The mechanistic understanding of film growth provided through experiments and electromagnetic simulations can further aid in adapting the microwave-assisted process to grow films of a broader range of materials on other microwave absorbing layers.

\section{Methods}

Preparation of substrates for film growth. Indium tin oxide (ITO) coated glass substrates with conductivity $\sigma \sim 10^{5} \mathrm{~S} / \mathrm{m}$ were purchased from Nanocs (New York, $\mathrm{NY})$ and cut into $\sim 1 \mathrm{~cm}$ by $\sim 1 \mathrm{~cm}$ square pieces. Lower conductivity $\left(\sigma \sim 10^{3} \mathrm{~S} / \mathrm{m}\right)$ ITO coated glass substrates were fabricated by a magnetron sputtering system equipped with a 3 -in. $\mathrm{In}_{2} \mathrm{O}_{3}: \mathrm{SnO}_{2}\left(10\right.$ wt. $\% \mathrm{SnO}_{2}$ ) sputtering target $(99.99 \%$ purity, Kurt J. Lesker company). The ITO films were grown by an RF power supply of $75 \mathrm{~W}$ with an Ar working pressure of 3 mTorr under a base pressure of $8.75 \times 10^{-5}$ Torr for $60 \mathrm{~min}$. In a typical process, the ITO layer was patterned by slow chemical etching in a mixture of $75 \%$ de-ionized water, $20 \% \mathrm{HCl}$, and $5 \% \mathrm{HNO}_{3}$. The substrates were then rinsed with de-ionized water and cleaned by successive sonication in aqueous detergent, de-ionized water, acetone, and isopropyl alcohol, and finally dried in flowing nitrogen. Deposition of $\mathrm{Al}$ films $\left(\sigma \sim 10^{7} \mathrm{~S} / \mathrm{m}\right)$ on cleaned glass substrates was achieved by a thermal evaporation process, carried out under a vacuum of $\sim 10^{-7}$ Torr using high purity aluminum wires (99.9995\%) hung on a tungsten wire basket. Thickness of the $\mathrm{Al}$ film was controlled to $150 \mathrm{~nm}$ by controlling the deposition current and time. Some experiments were also performed on ITO-coated PET substrates from Nanocs (conductivity $\sigma \sim 10^{5} \mathrm{~S} / \mathrm{m}$ ). 
Microwave-assisted $\mathrm{TiO}_{2}$ film growth. A sol-gel solution based on tetrabutyl orthotitanate was prepared as has been previously reported ${ }^{14}$. Generally, $5 \mathrm{~mL}$ of the sol-gel was combined with $20 \mathrm{~mL}$ of tetraethylene glycol (TEG) in $80 \mathrm{~mL}$ quartz vessels designed for a $2.45 \mathrm{GHz}$ Anton Paar Synthos 3000 microwave reactor. The substrates were placed inside custom designed glass baskets which were suspended from the top of the vessels. Most experiments were performed with the substrates aligned vertically, but a few experiments were also performed with the substrates aligned horizontally. Stir bars were added to ensure efficient mixing and the vessels were sealed. The vessels were then positioned on a rotor (up to 8 vessels at a time), which was spun on a turntable such that the microwave irradiation would be uniform. The ramp rate, temperature, and reaction time were varied. The temperature was measured with infrared sensors on each vessel. After the reaction, the solutions were cooled to room temperature, and the resulting $\mathrm{TiO}_{2}$ films were washed and sonicated in de-ionized water, acetone, and ethanol to remove impurities like TEG.

SiC vessel experiment. An Anton Paar Monowave microwave was also used in this work for the results in Fig. 7. In $10 \mathrm{~mL} \mathrm{SiC}$ and glass vessels, an ITO-coated glass substrate was suspended from the top of the vessels with a custom made basket tied to the vessel cap by Teflon tape. The reaction temperature was measured directly within the growth solution via a thermometer inside of an immersion tube. The thermometer was located directly behind the glass substrate in these reactions. The temperature was ramped to $160^{\circ} \mathrm{C}$ in approximately $3 \mathrm{~min}$ and held for $60 \mathrm{~min}$. More details are given in Supplementary Section 5

Microwave-grown film characterization. Glancing incidence X-ray diffraction (GIXRD) experiments were performed with a Rigaku Ultima IV diffractometer operating in parallel beam (PB) mode at $40 \mathrm{kV}$ and $44 \mathrm{~mA}$ using $\mathrm{CuK} \alpha$ radiation $(\lambda$ $=1.54 \AA$ ) in the $2 \theta$ range of 20 to $80^{\circ}$ at a step of $0.02^{\circ}$ and a glancing angle of $0.5^{\circ}$. Scanning electron micrographs (SEM) were recorded using an FEI Quanta 650 SEM. The film thicknesses were obtained by cross-sectional SEM imaging. Atomic force microscopy (AFM) images were taken on an Agilent 5500 AFM. Conductive AFM (C-AFM) imaging was carried out in contact mode using a conductive (aluminum coated silicon) tip and a $3 \mathrm{~V}$ applied electrical bias to the sample surface. Circuitry inside the AFM can thus measure the current that flows when the tip comes in contact with the surface of the sample. Here, we use C-AFM images to simultaneously map topography of the microwave-grown $\mathrm{TiO}_{2}$ films along with areas of varying conductivity. Low magnification scanning transmission electron microscopy (STEM) images were obtained on Hitachi S-5500. High resolution transmission electron microscopy (TEM) images were obtained on a JEOL 2010F, operating under $200 \mathrm{keV}$. For STEM and TEM analysis, a $5 \mathrm{~mm}$ x $5 \mathrm{~mm}$ section of the $\mathrm{TiO}_{2}$ film was scraped off the ITO substrates, and then dispersed in isopropyl alcohol by sonication for $5 \mathrm{~min}$. Small aliquots of this suspension were collected on a lacy carbon grid and dried overnight under vacuum. The Raman spectra acquired by the Renishaw inVia Raman Microscope were performed with $50 \%$ of the $50 \mathrm{~mW} 514.5 \mathrm{~nm}$ laser excitation at a 50X objective. Optical micrographs were obtained with a Nikon Eclipse ME600 optical microscope. Conductivity of films (ITO, metal) were measured using a fourprobe conductivity measurement system comprising of a Lucas Signatone four-point probe head and stand, combined with a Keithley 2400 source meter.

Electromagnetic simulation of microwave-assisted film growth. Electromagnetic simulations were performed using a fast Fourier transform accelerated frequencydomain integro-differential equation solver that models the glass substrate as an ideal lossless dielectric, the ITO layer as an imperfect (but good) conductor, the liquid solution as a lossy dielectric, the quartz vessel as another lossless dielectric, and the microwave reactor as a cavity containing a rotor with perfectly conducting walls. A hybrid volume-surface integral equation formulation was employed to compute the microwave fields at various positions of the rotor and these fields were processed to obtain the energy absorbed at various positions in the model; the governing equations and their numerical solution are detailed in Supplementary Section 4.

1. Turnbull, D. Kinetics of heterogeneous nucleation. J. Chem. Phys. 18, 198-203 (1950).

2. Venables, J. A., Spliller, G. D. T. \& Hanbucken, M. Nucleation and growth of thin films. Rep. Prog. Phys. 47, 399-459 (1984).

3. Venables, J. A. Rate equation approaches to thin film nucleation kinetics. Phil. Mag. 27, 697-738 (1973)

4. Vigil, E. et al. $\mathrm{TiO}_{2}$ thin film deposition from solution using microwave heating. Thin Solid Films 365, 12-18 (2000).

5. Ayllon, J. A. et al. J. Preparation of anatase powders from fluorine-complexed titanium(IV) aqueous solution using microwave irradiation. J. Mater. Chem. 10, 1911-1914 (2000)

6. Baghbanzadeh, M., Carbone, L., Cozzoli, P. D. \& Kappe, C. O. Microwave-assisted synthesis of colloidal inorganic nanocrystals. Angew. Chem. Int. Ed. 50, 11312-11359 (2011).

7. De la Hoz, A., Diaz-Ortiz, A. \& Moreno, A. Microwaves in organic synthesis. Thermal and non-thermal microwave effects. Chem. Soc. Rev. 34, 164-178 (2005).
8. Bilecka, I., Elser, P. \& Niederberger, M. Kinetic and thermodynamic aspects in the microwave-assisted synthesis of $\mathrm{ZnO}$ nanoparticles in benzyl alcohol. ACS Nano. 3, 467-477 (2009).

9. Rao, K. J., Vaidhyanathan, B., Ganguli, M. \& Ramakrishnan, P. A. Synthesis of inorganic solids using microwaves. Chem. Mater. 11, 882-895 (1999).

10. Conner, W. C. \& Tompsett, G. A. How could and do microwaves influence chemistry at interfaces? J. Phys. Chem. B 112, 2110-2118 (2008).

11. Diebold, U. The surface science of titanium dioxide. Surface Science Reports 48 , 53-229 (2003).

12. Niederberger, M. \& Pinna, N. Metal oxide nanoparticles in organic solventssynthesis formation, assembly and application Chap. 8 (Springer, London, 2009).

13. Fernandez-Garcia, M., Martinez-Arias, A., Hanson, J. C. \& Rodriguez, J. A. Nanostructured oxides in chemistry: characterization and properties. Chem. Rev. 104, 4063-4104 (2004).

14. Xue, H. et al. $\mathrm{TiO}_{2}$ based metal-semiconductor-metal ultraviolet photodetectors. Appl. Phys. Lett. 90, 201118-1-201118-3 (2007).

15. Dutta, P. K. et al. Interaction of CO with anatase surfaces at high temperatures: optimization of a CO sensor. J. Phys. Chem. B 103, 4412-4422 (1999).

16. O'Regan, B. \& Gratzel, M. A low-cost, high-efficiency solar cell based on dyesensitized colloidal $\mathrm{TiO}_{2}$ films. Nature 353, 737-740 (1991).

17. Fujishima, A. \& Honda, K. Electrochemical photolysis of water at a semiconductor electrode. Nature 238, 37-38 (1972).

18. Coakley, K. M. \& McGehee, M. D. Photovoltaic cells made from conjugated polymers infiltrated into mesoporous titania. Appl. Phys. Lett. 83, 3380-3382 (2003).

19. Natarajan, C., Fukunaga, N. \& Nogami, G. Titanium dioxide thin film deposited by spray pyrolisis of aqueous solution. Thin Solid Films 322, 6-8 (1998).

20. Djenizian, T., Hanzu, I. \& Knauth, P. Nanostructured negative electrodes based on titania for Li-ion microbatteries. J. Mater. Chem. 21, 9925-9937 (2011).

21. Montaudo, G., Puglisi, C. \& Samperi, F. Primary thermal degradation mechanisms of PET and PBT. Polym Degrad Stab 42, 13-28 (1993).

22. Ohsaka, T., Izumi, F. \& Fujiki, Y. Raman spectrum of anatase $\mathrm{TiO}_{2}$. J. Raman Spectrosc. 7, 321-324 (1978).

23. Meixner, J. The behavior of electromagnetic fields at edges. IEEE Trans. Antennas Propagat. 20, 442-446 (1972).

24. Bladel, J. V. Singular electromagnetic fields and sources. IEEE Press Series on Electromagnetic Wave Theory 39, 1312-1320 (1991).

25. Flugge, H. et al. Microwave annealing of polymer solar cells with various transparent anode materials. Appl. Phys. Lett. 97, 123306-1-123306-3 (2010)

26. Obermayer, D., Gutmann, B. \& Kappe, C. O. Microwave chemistry in silicon carbide reaction vials: separating thermal from nonthermal effects. Angewandte Chemie International Edition 121, 8471-8474 (2009).

27. Fini, A. \& Breccia, A. Chemistry by microwaves. Pure Appl. Chem. 71, 573-579 (1999).

28. Gou, L., Chipara, M. \& Zaleski, J. M. Convenient, rapid synthesis of Ag nanowires. Chem. Mater. 19, 1755-1760 (2007)

\section{Acknowledgements}

The synthesis and XRD characterization work was supported by the U.S. Department of Energy, Office of Basic Energy Sciences, Division of Materials Science and Engineering under Award Number DE-SC0005397. The spectroscopic, microscopic, and electrical characterization work was supported by the Energy Frontier Research Center funded by the U.S. Department of Energy, Office of Science, Office of Basic Energy Sciences under Award Number DE-SC0001091. The modeling work was supported in part by the National Science Foundation Grant OCI-0904907. The authors thank Dr. Joshua Bolinger for assistance with electron beam lithography on ITO films and Dr. Wei Li for assistance with STEM and TEM imaging. The authors acknowledge the Texas Advanced Computing Center (TACC) at the University of Texas at Austin for providing HPC and visualization resources that contributed to the research results reported in this paper.

\section{Author contributions}

Katharine Harrison, Reeja Jayan, and Chih-Liang Wang carried out the synthesis and characterization work under the supervision of Professor Arumugam Manthiram. Kai Yang carried out the modeling work under the supervision of Professor Ali Yilmaz.

\section{Additional information}

Supplementary information accompanies this paper at http://www.nature.com/ scientificreports

Competing financial interests: The authors declare no competing financial interests.

License: This work is licensed under a Creative Commons Attribution-

NonCommercial-NoDerivs 3.0 Unported License. To view a copy of this license, visit http:// creativecommons.org/licenses/by-nc-nd/3.0/

How to cite this article: Reeja-Jayan, B. et al. Microwave-assisted Low-temperature Growth of Thin Films in Solution. Sci. Rep. 2, 1003; DOI:10.1038/srep01003 (2012). 